\title{
CONSTRUCTION OF COMPACTIFICATIONS USING ESSENTIAL SEMILATTICE HOMOMORPHISMS
}

\author{
A. CATERINO, G. D. FAULKNER, AND M. C. VIPERA
}

(Communicated by James E. West)

\begin{abstract}
In this paper we introduce a new method for constructing compactifications of a locally compact space. The method generalizes the notion of a singular compactification to a much larger collection of compactifications, which we call ESH-compactifications. In particular, if $X$ is paracompact, or realcompact, then $\beta X$ is of this form. We also establish conditions that ensure an ESH-compactification is a singular or weakly singular compactification.
\end{abstract}

\section{INTRODUCTION}

Let $X$ be a locally compact Hausdorff space and $K$ a compact Hausdorff space. A function $f: X \rightarrow K$ is said to be singular if for each nonempty open $U \subset K$, the set $f^{-1}(U)$ is not relatively compact. Singular mappings have been extensively used to study compactifications of locally compact spaces [1, $4,5,9]$. In particular, a compactification of $X$ having $K$ as a remainder may be constructed by taking as a basis in $X \cup K$ the open sets of $X$ together with sets of the form $U \cup\left(f^{-1}(U) \backslash F\right)$, where $U$ is an arbitrary open set in $K$ and $F$ is an arbitrary compact subset of $X$. This compactification is usually denoted $X \cup_{f} K$. It is clear that many compactifications cannot arise in this manner. In particular, $\beta X$ is very infrequently of this form [6,7]. This paper is concerned with a generalization of this construction that has much wider applicability. To motivate the definition, let us consider the construction associated with a singular mapping. Let $\mathscr{N}$ be the set of nonrelatively compact subsets of $X$ together with $\varnothing . \mathscr{N}$ is an upper semilattice. In the construction of $X \cup_{f} K$, we may as well have chosen to use sets $U \subset K$ from some basis $\mathscr{B}$. Also, without loss of generality, we may assume that $\mathscr{B}$ is closed under finite unions so that it is also an upper semilattice. This, of course, implies that $K \in \mathscr{B}$. The mapping that takes open sets in $K$ to open sets in $X$, defined by $\pi(U)=f^{-1}(U)$, is a lattice homomorphism from the topology on $K$ to the topology on $X$ that carries $\mathscr{B}$ into $\mathscr{N}$. This is the motivating structure for what follows. We will see that it is much stronger than is required and that the relaxed definition yields a much richer collection of compactifications. It is in

Received by the editors October 20, 1989 and, in revised form, March 25, 1991; the contents of this paper were presented by G. D. Faulkner to the annual AMS meeting, Atlanta, GA, January 1990.

1991 Mathematics Subject Classification. Primary 54D35, 54D40, 54C10. 
fact possible, and as yet unknown to us, that all compactifications arise in this manner. It is true, as we will observe later, that this class of compactifications is distinct from the collection of Wallman compactifications, to which they bear some superficial resemblance. All topological spaces in this paper are assumed to be Hausdorff and locally compact. The symmetric difference of sets $A$ and $B$ will be denoted $A \triangle B$.

\section{MAIN DEFINITION}

Let $X$ be a locally compact noncompact space, and let $\mathscr{N}$ be the set of nonrelatively compact open subsets of $X$ together with $\varnothing$. Let $K$ be a compact space, and let $\mathscr{B}$ be a basis for the open subsets of $K$. Suppose that $\mathscr{B}$ is closed with respect to finite unions, which, of course, implies that $K \in \mathscr{B}$. Let $\pi: \mathscr{B} \rightarrow \mathscr{N}$ such that $\pi(U) \neq \varnothing$ for every $U \neq \varnothing$. We say that $\pi$ is an essential semilattice homomorphism (ESH for short) if it satisfies the following conditions:

ESH1. $X \backslash \pi(K)$ is compact.

ESH2. $\pi(U \cup V) \triangle(\pi(U) \cup \pi(V))$ is relatively compact, $\forall U, V \in \mathscr{B}$.

ESH3. If $U, V \in \mathscr{B}$ and $\bar{U} \cap \bar{V}=\varnothing$, then $\pi(U) \cap \pi(V)$ is relatively compact.

A lattice homomorphism has all these properties and more. The use of the word essential is to indicate that the properties, which are shared by a lattice homomorphism, hold except on a negligible set. In the study of compactifications of a space $X$, the compact subsets of $X$ are negligible. We observe that, if $\pi$ is an ESH and $\varnothing \in \mathscr{B}$, then $\pi(\varnothing)$ is relatively compact, by ESH3, and so $\pi(\varnothing)=\varnothing$. Moreover, it follows from ESH2 that if $U, V \in \mathscr{B}, U \subset V$, then $\pi(U) \backslash \pi(V)$ is relatively compact. If $\pi$ is an ESH, there is a natural way to define a topology on the disjoint union of $X$ and $K$. Let

$$
\mathscr{S}=\{W \subset X \cup K \mid W=U \cup(\pi(U) \backslash F), U \in \mathscr{B}, F \subset X, F \text { compact }\},
$$

and let $\mathscr{A}=\mathscr{S} \cup \mathscr{T}$, where $\mathscr{T}$ is the topology of $X . \mathscr{A}$ is a basis for a topology on $X \cup K$. The only thing in need of proof is that, for $W_{1}, W_{2} \in \mathscr{S}$ and for each $y \in W_{1} \cap W_{2}$, with $y \in K$, there exists $W_{3} \in \mathscr{A}$ such that $y \in$ $W_{3} \subset W_{1} \cap W_{2}$. To this end, let $W_{1}=U_{1} \cup\left(\pi\left(U_{1}\right) \backslash F_{1}\right), W_{2}=U_{2} \cup\left(\pi\left(U_{2}\right) \backslash F_{2}\right)$, and let $U_{3} \in \mathscr{B}$ be such that $y \in U_{3} \subset U_{1} \cap U_{2}$. Both $\pi\left(U_{3}\right) \backslash \pi\left(U_{1}\right)$ and $\pi\left(U_{3}\right) \backslash \pi\left(U_{2}\right)$ are relatively compact. This implies $\pi\left(U_{3}\right) \subset \pi\left(U_{1}\right) \cup G_{1}, \pi\left(U_{3}\right) \subset$ $\pi\left(U_{2}\right) \cup G_{2}$, where $G_{1}, G_{2}$ are compact subsets of $X$. Put $F=F_{1} \cup F_{2} \cup G_{1} \cup G_{2}$ and $W_{3}=U_{3} \cup\left(\pi\left(U_{3}\right) \backslash F\right)$. It is clear in this case that one has $y \in W_{3} \subset W_{1} \cap W_{2}$ and $W_{3} \in \mathscr{S}$. We denote by $X \cup_{\pi} K$ the set $X \cup K$, endowed with the topology generated by $\mathscr{A}$. This topology induces the original topologies on the subspaces $X$ and $K$. We will later need the following:

Remark 1 . It is easy to see that, for every basis $\mathscr{V}$ of $X, \mathscr{S} \cup \mathscr{V}$ is a basis for $X \cup_{\pi} K$.

\section{RESULTS}

Proposition 1. $X \cup_{\pi} K$ is a compact Hausdorff space having $X$ as a dense subspace.

Proof. First we prove that $X \cup_{\pi} K$ is Hausdorff. We need only consider two nontrivial cases. For the first suppose $x \in X$ and $y \in K$. Let $U \in \mathscr{B}$ be such 
that $y \in U$ and let $F$ be a compact neighborhood of $x$ in $X$. It follows that $F$ and $U \cup(\pi(U) \backslash F)$ are disjoint neighborhoods, in $X \cup_{\pi} K$, of $x$ and $y$, respectively. Next suppose $x, y \in K$ and let $U, V$ be elements of $\mathscr{B}$ such that $x \in U, y \in V$, and $\bar{U} \cap \bar{V}=\varnothing$. By the definition, there exists a compact subset $F_{1}$ of $X$ such that $\pi(U) \cap \pi(V) \subset F_{1}$. Then $U \cup\left(\pi(U) \backslash F_{1}\right)$ and $V \cup \pi(V)$ are disjoint neighbourhoods, in $X \cup_{\pi} K$, of $x$ and $y$, respectively. In order to prove that $X \cup_{\pi} K$ is compact, let $\mathscr{C} \subset \mathscr{A}$ be a cover of $X \cup_{\pi} K$ and let $\mathscr{D}=\left\{W_{i}\right\}$ be a finite subfamily of $\mathscr{C}$ that covers $K$. We can suppose that each $W_{i}$ is of the form $U_{i} \cup\left(\pi\left(U_{i}\right) \backslash F_{i}\right)$, where $U_{i} \in \mathscr{B}$ and $F_{i}$ is a compact subset of $X$. Let $F=\bigcup F_{i}$ and $S=X \backslash \pi(K)$. Since $K=\bigcup U_{i}$, it follows by ESH 2 that there exists a compact $T \subset X$ such that $\pi(K) \backslash T \subset \bigcup \pi\left(U_{i}\right)$. Thus one has

$$
X \backslash(F \cup T \cup S)=\pi(K) \backslash(F \cup T) \subset\left(\bigcup \pi\left(U_{i}\right)\right) \backslash F \subset \bigcup\left(\pi\left(U_{i}\right) \backslash F_{i}\right) .
$$

Thus $\mathscr{D}$ covers all of $X \cup_{\pi} K$, except $F \cup T \cup S$, which is compact. It follows that we can find a finite subcover of $\mathscr{C}$.

In view of the above proposition, an ESH can be used to obtain a compactification of $X$. A compactification of $X$ is said to be an ESH-compactification if it is equivalent to $X \cup_{\pi} K$, for suitable $K$ and $\pi$.

Example 1. As previously indicated, every singular compactification $X \cup_{f} K$ is an ESH-compactification. If $\mathscr{B}$ is the topology of $K$, we can put $\pi(U)=$ $f^{-1}(U)$, for each $U \in \mathscr{B}$. Now singular compactifications are characterized by the existence of a retraction $r: \alpha X \rightarrow \alpha X \backslash X$. In fact, the singular map $f$ extends to a retraction. In [7] weakly singular compactifications, which are compactifications for which the remainder is a neighborhood retract, are studied. The two-point compactification of the real numbers is an example of a weakly singular compactification that is not singular. In fact, all compactifications with finite remainders are weakly singular. In this context, if $r: W \rightarrow \alpha X \backslash X$, where $W=\alpha X \backslash F, F$ a compact subset of $X$, and $f=r_{\mid X \cap W}$, then, as before, $\pi(U)=f^{-1}(U)$ is an essential semilattice homomorphism that makes $\alpha X$ into an ESH-compactification.

Example 2. The class of ESH-compactifications is distinct from the class of Wallman compactifications. Let $\alpha X$ be a compactification that is not Wallman ( $X$ need not be locally compact). We will construct a singular compactification of a discrete space that is not Wallman. We use, with some modification, the construction in [3, pp. 95-96]. Let $g$ be a function from a discrete space $D$ onto $X$ such that $\forall x \in X \quad g^{-1}(x)$ is an infinite set. Let $f: D \rightarrow \alpha X$ be the composition of $g$ and the inclusion of $X$ into $\alpha X$. Then $f$ is singular. Let $F: D \cup_{f} \alpha X \rightarrow \alpha X$ be defined by $\left.F\right|_{D}=f$ and $\left.F\right|_{\alpha X}=1_{\alpha X} . \quad F$ is clearly continuous and satisfies the hypotheses of Lemma 8.5 in [3]. Therefore, if $D \cup_{f} \alpha X$ was a Wallman compactification, then $\alpha X$ would be as well. But $D \cup_{f} \alpha X$, being singular, is an ESH-compactification.

It is typically difficult to prove that a given compactification is an ESHcompactification from first principles. The following lemma provides a means for showing that a compactification arises in this manner without the necessity of explicitly constructing the mapping $\pi$. 
Lemma 2. Let $\alpha X$ be a compactification of $X$. Then $\alpha X$ is an ESH-compactification if and only if there exists a basis $\mathscr{U}$ (for the open subsets) of $\alpha X$ that is closed with respect to finite unions and satisfies the following conditions:

(C1) if $W, W^{\prime} \in \mathscr{U}$ and $W \cap(\alpha X \backslash X)=W^{\prime} \cap(\alpha X \backslash X)$, then $W \triangle W^{\prime}$ is a relatively compact subset of $X$

(C2) if $W, W^{\prime} \in \mathscr{U}$ and $\overline{W \cap(\alpha X \backslash X)} \cap \overline{W^{\prime} \cap(\alpha X \backslash X)}=\varnothing$, then $W \cap W^{\prime}$ is relatively compact in $X$.

Proof. Let $\alpha X$ be an ESH-compactification. Then there exists a basis $\mathscr{B}$ of $K=\alpha X \backslash X$ and an ESH $\pi: \mathscr{B} \rightarrow \mathscr{N}$ such that $\alpha X=X \cup_{\pi} K$. If we denote by $\mathscr{R}$ the set of relatively compact open subsets of $X$, then, in view of Remark $1, \mathscr{W}=\mathscr{S} \cup \mathscr{R}$ is a basis of $X \cup_{\pi} K$. Let $\mathscr{U}$ be the set of finite unions of elements of $\mathscr{W}$. We want to prove that $\mathscr{U}$ satisfies conditions $(\mathrm{C} 1)$ and $(\mathrm{C} 2)$. Let $W, W^{\prime} \in \mathscr{U}$. Then one has

$$
W=\left(\bigcup_{i=1}^{r} U_{i}\right) \cup\left(\bigcup_{i=1}^{r}\left(\pi\left(U_{i}\right) \backslash F_{i}\right)\right) \cup T
$$

and

$$
W^{\prime}=\left(\bigcup_{j=1}^{s} V_{j}\right) \cup\left(\bigcup_{j=1}^{s}\left(\pi\left(V_{j}\right) \backslash G_{j}\right)\right) \cup T^{\prime},
$$

where the $U_{i}$ 's, $V_{j}$ 's are elements of $\mathscr{B}$, the $F_{i}$ 's, $G_{j}$ 's are compact subsets of $X$, and $T, T^{\prime}$ are relatively compact open subsets of $X$. The hypothesis $W \cap K=W^{\prime} \cap K$ can be written $\bigcup_{i=1}^{r} U_{i}=\bigcup_{j=1}^{s} V_{j}$. This implies

$$
\pi\left(\bigcup_{i=1}^{r} U_{i}\right) \triangle \pi\left(\bigcup_{j=1}^{s} V_{j}\right)=\varnothing
$$

Thus

$$
\begin{aligned}
& \left(\bigcup_{i=1}^{r} \pi\left(U_{i}\right)\right) \triangle\left(\bigcup_{j=1}^{s} \pi\left(V_{j}\right)\right) \\
& \quad=\left(\left(\bigcup_{i=1}^{r} \pi\left(U_{i}\right)\right) \triangle \pi\left(\bigcup_{i=1}^{r} U_{i}\right)\right) \triangle\left(\pi\left(\bigcup_{j=1}^{s} V_{j}\right) \triangle\left(\bigcup_{j=1}^{s} \pi\left(V_{j}\right)\right)\right),
\end{aligned}
$$

which is relatively compact, by ESH2. Thus $W \triangle W^{\prime}$ is a relatively compact subset of $X$. This proves $(\mathrm{C} 1)$.

Next, suppose $\overline{W \cap K} \cap \overline{W^{\prime} \cap K}=\varnothing$. Then one has $\left(\bigcup_{i=1}^{r} \bar{U}_{i}\right) \cap\left(\bigcup_{j=1}^{s} \bar{V}_{j}\right)=$ $\varnothing$, which implies $\bar{U}_{i} \cap \bar{V}_{j}=\varnothing$, for each $i, j$. Therefore, by ESH3, $\pi\left(U_{i}\right) \cap$ 
$\pi\left(V_{j}\right)$ is relatively compact for each $i, j$. Therefore

$$
\begin{aligned}
W \cap W^{\prime} & \subset\left(\left(\bigcup_{i=1}^{r} \pi\left(U_{i}\right)\right) \cup T\right) \cap\left(\left(\bigcup_{j=1}^{s} \pi\left(V_{j}\right)\right) \cup T^{\prime}\right) \\
\subset & \left(\left(\bigcup_{i=1}^{r} \pi\left(U_{i}\right)\right) \cap\left(\bigcup_{j=1}^{r} \pi\left(V_{j}\right)\right)\right) \cup\left(T \cup T^{\prime}\right) \\
= & \left(\bigcup_{i, j}\left(\pi\left(U_{i}\right) \cap \pi\left(V_{j}\right)\right)\right) \cup\left(T \cup T^{\prime}\right),
\end{aligned}
$$

which is a relatively compact subset of $X$. This proves (C2). Conversely, let $\mathscr{B}=\{U \subset K \mid \exists W \in \mathscr{U}$ such that $U=W \cap K\}$. Clearly $\mathscr{B}$ is closed with respect to finite unions. One can easily see that if $W \in \mathscr{U}$ and $W \cap K \neq$ $\varnothing$, then $W \cap X$ is not relatively compact in $X$. Thus, using the Axiom of Choice, we can define a map $\pi$ from $\mathscr{B}$ to $\mathscr{N}$ in the following way: we put $\pi(\varnothing)=\varnothing$; if $U \neq \varnothing$, we choose $W \in \mathscr{U}$ such that $U=W \cap K$ and we put $\pi(U)=W \cap X$. $\pi$, so defined, is an ESH. We see this as follows. To prove ESH1, we observe that $\alpha X \in \mathscr{U}$, hence, for $W \in \mathscr{U}, W \cap K=K$ implies, by (C1), that $\alpha X \Delta W=\alpha X \backslash W=X \backslash(W \cap X)$ is a compact subset of $X$. Thus $X \backslash \pi(K)$ is compact. Let $U_{1}, U_{2} \in \mathscr{B}$. Put $\pi\left(U_{1} \cup U_{2}\right)=W \cap X$, where $W \cap K=U_{1} \cup U_{2}$, and put $\pi\left(U_{i}\right)=W_{i} \cap X$, where $W_{i} \cap K=U_{i}, i=1,2$. One has $\left(W_{1} \cup W_{2}\right) \cap K=U_{1} \cup U_{2}$. Therefore, condition (C1) implies that $\left(W_{1} \cup W_{2}\right) \triangle W$ is a relatively compact subset of $X$. Clearly one has

$$
\begin{aligned}
\left(W_{1} \cup W_{2}\right) \triangle W & =\left(\left(W_{1} \cup W_{2}\right) \cap X\right) \triangle(W \cap X) \\
& =\left(\pi\left(U_{1}\right) \cup \pi\left(U_{2}\right)\right) \triangle \pi\left(U_{1} \cup U_{2}\right),
\end{aligned}
$$

and this proves ESH2. Now, let $U_{1}, U_{2} \in \mathscr{B}$ be such that $\bar{U}_{1} \cap \bar{U}_{2}=\varnothing$. Let $\pi\left(U_{i}\right)=W_{i} \cap X$, where $W_{i} \cap K=U_{i}, i=1,2$. Since $\left(\overline{W_{1} \cap K}\right) \cap\left(\overline{W_{2} \cap K}\right)=\varnothing$, by $(\mathrm{C} 2), W_{1} \cap W_{2}=\pi\left(U_{1}\right) \cap \pi\left(U_{2}\right)$ is a relatively compact subset of $X$. This proves ESH3. Finally, it is easy to see that $X \cup_{\pi} K$ is equivalent to $\alpha X$. In fact, let $U \in \mathscr{B}$ and let $\pi(U)=W \cap X$, where $W \cap K=U$. If $F$ is a compact subset of $X$, then one has $U \cup(\pi(U) \backslash F)=W \backslash F$, which is clearly open in $\alpha X$.

Corollary 3. Let $\alpha X$ be a compactification of $X$. Suppose that there exists a basis $\mathscr{U}$ (for the open subsets) of $\alpha X$ that is closed with respect to finite unions and intersections and satisfies condition (C1). Then $\alpha X$ is an ESHcompactification.

Proof. If $\mathscr{U}$ is closed with respect to finite intersections, then $\varnothing \in \mathscr{U}$ and it is easy to see that $(\mathrm{C} 1)$ implies $(\mathrm{C} 2)$.

Theorem 4. If $\alpha X \backslash X$ is 0-dimensional, then $\alpha X$ is an ESH-compactification. Proof. Let $K=\alpha X \backslash X$ and let $\mathscr{F}$ be the family of the closed subsets of $\alpha X$ such that every point $x \in F \cap K$ is in the interior of $F . \mathscr{F}$ is closed with respect finite unions and intersections. We want to prove that $\mathscr{F}$ is a basis for the closed subsets of $\alpha X$, that is, for a given closed subset $G$ of $\alpha X$ and for $x \notin G$, there exists $F \in \mathscr{F}$ such that $x \notin F$ and $G \subset F$. If $x \in X$, we can 
choose $F=\alpha X \backslash W$, where $W \subset X$ is a relatively compact open neighbourhood of $x$ with $W \cap G=\varnothing$. Now let $x \in K$. Let $A$ be an open and closed neighbourhood of $x$ in $K$ such that $A \cap G=\varnothing$ and let $B=K \backslash A$. Since $A$ and $B$ are disjoint closed subsets of $\alpha X$, there exist disjoint open subsets $U_{1}, U_{2}$ of $\alpha X$ with $A \subset U_{1}$ and $B \subset U_{2}$. Put $F=G \cup \mathrm{Cl}_{\alpha X}\left(U_{2}\right)$. One has $x \notin F, F \cap K=B \subset U_{2} \subset F$, hence $F \in \mathscr{F}$.

Now suppose $F_{1}, F_{2} \in \mathscr{F}$ and suppose $F_{1} \cap K=F_{2} \cap K=S$. By definition of $\mathscr{F}$, there exist $V_{1}, V_{2}$ open subsets of $\alpha X$ such that $S \subset V_{i} \subset F_{i}, i=1,2$. Then $F_{1} \Delta F_{2}=\left(F_{1} \cup F_{2}\right) \backslash\left(F_{1} \cap F_{2}\right) \subset\left(F_{1} \cup F_{2}\right) \backslash\left(V_{1} \cap V_{2}\right)$, which is contained in $X$ and is a closed subset of $\alpha X$, hence a compact. Let $\mathscr{B}=\{\alpha X \backslash F \mid F \in \mathscr{F}\}$. The set-theoretic identity $\left(\alpha X \backslash F_{1}\right) \triangle\left(\alpha X \backslash F_{2}\right)=F_{1} \triangle F_{2}$ implies that $\mathscr{B}$ satisfies the hypothesis of Corollary 3.

Theorem 5. Every compactification of $X$ is the supremum of a family of ESHcompactifications.

Proof. Let $\alpha X$ be a compactification of $X$. If $\alpha X \backslash X$ is not scattered, then, by [2], $\alpha X$ is the supremum of a family of singular compactifications. On the other hand, if $\alpha X \backslash X$ is scattered, it is 0-dimensional, and hence, by Theorem 4, $\alpha X$ is an ESH-compactification.

We will show now that two broad and important collections of compactifications are in fact ESH-compactifications. First we have

\section{Theorem 6. If $X$ is paracompact, then $\beta X$ is an ESH-compactification.}

Proof. Since a paracompact space is normal, $\beta X$ coincides with the Wallman compactification, hence the collection $\left\{\mathrm{Cl}_{\beta X}(F) \mid F\right.$ is a closed subset of $\left.X\right\}$ is a basis for closed subsets of $\beta X$. Moreover, if $F$ and $G$ are closed subsets of $X$, then one has $\mathrm{Cl}_{\beta X}(F \cap G)=\mathrm{Cl}_{\beta X}(F) \cap \mathrm{Cl}_{\beta X}(G)$ [12]. Thus $\mathscr{U}=$ $\left\{\beta X \backslash \mathrm{Cl}_{\beta X}(F) \mid F\right.$ is a closed subset of $\left.X\right\}$ is a basis for the open subsets of $\beta X$ and it is closed with respect to finite unions and intersections. We have only to prove that $\mathscr{U}$ satisfies condition $(\mathrm{C} 1)$. Let $W, W^{\prime} \in \mathscr{U}$ be such that $W \cap K=W^{\prime} \cap K$, where $K=\beta X \backslash X$. We want to prove that $W \triangle W^{\prime}$ is relatively compact in $X$. Let $F, G$ be closed subsets of $X$ such that $W=$ $\beta X \backslash \mathrm{Cl}_{\beta X}(F), W^{\prime}=\beta X \backslash \mathrm{Cl}_{\beta X}(G)$. Then one has $\mathrm{Cl}_{\beta X}(F) \cap K=\mathrm{Cl}_{\beta X}(G) \cap K$. Moreover, one has $W \triangle W^{\prime}=(X \backslash F) \triangle(X \backslash G)=F \triangle G$. Thus it suffices to prove that $F \triangle G$ is relatively compact in $X$. First suppose $F \subset G$, so that $F \triangle G=G \backslash F$. Suppose $G \backslash F$ is not relatively compact. We will construct a closed nonrelatively compact subset, $G_{1}$, of $G \backslash F$. Since $X$ is paracompact, it can be written as a topological sum $\sum_{\alpha \in \Gamma} X_{\alpha}$, where each of $X_{\alpha}$ is $\sigma$-compact. Suppose that $G \backslash F$ meets infinitely many of the $X_{\alpha}$ 's, say $(G \backslash F) \cap X_{\lambda} \neq \varnothing$ for $\lambda \in \Gamma^{\prime} \subset \Gamma$, where $\Gamma^{\prime}$ is an infinite set. Let $G_{1}=\left\{x_{\lambda}\right\}$ be constructed by choosing a single point from each of these intersections. $\left\{x_{\lambda}\right\}$ is clearly infinite, closed, and discrete. If $G \backslash F$ meets only finitely many $X_{\alpha}$ then it must meet at least one $X_{\alpha_{0}}$ in a nonrelatively compact set $H$. Now $X_{\alpha_{0}}$ can be written as the union of countably many open sets, each having compact closure, say $X_{\alpha_{0}}=\bigcup_{i=1}^{\infty} U_{i}$. For each $n \in \mathbf{N}$, choose $x_{n} \in H \backslash \bigcup_{i=1}^{n-1} U_{i}$. Let $G_{1}=\left\{x_{n}\right\}$. This set is again infinite, closed, and discrete. By the construction, $F$ and $G_{1}$ are disjoint closed subsets of $X$, and this implies $\mathrm{Cl}_{\beta X}(F) \cap \mathrm{Cl}_{\beta X}\left(G_{1}\right)=\varnothing$. However, $G_{1}$ must have a limit point $y \in \beta X \backslash X$. But $y \in \mathrm{Cl}_{\beta X}\left(G_{1}\right) \cap K \subset$ $\mathrm{Cl}_{\beta X}(G) \cap K=\mathrm{Cl}_{\beta X}(F) \cap K$, which is a contradiction. 
Now we want to prove that $F \triangle G$ is relatively compact in the general case. One has

$$
\begin{aligned}
\mathrm{Cl}_{\beta X}(F \cap G) \cap K & =\left(\mathrm{Cl}_{\beta X}(F) \cap \mathrm{Cl}_{\beta X}(G)\right) \cap K \\
& =\left(\mathrm{Cl}_{\beta X}(F) \cap K\right) \cap\left(\mathrm{Cl}_{\beta X}(G) \cap K\right)=\mathrm{Cl}_{\beta X}(F) \cap K .
\end{aligned}
$$

Similarly, we can prove $\mathrm{Cl}_{\beta X}(F \cup G) \cap K=\mathrm{Cl}_{\beta X}(F) \cap K$. By an application of the previous argument to $F \cap G$ and $F \cup G$, we see that $(F \cup G) \backslash(F \cap G)=F \triangle G$ is relatively compact. This completes the proof.

The following theorem was provided by the referee.

Theorem 7. If $X$ is realcompact then $\beta X$ is an ESH-compactification.

Proof. Let $\mathscr{U}=\left\{\beta X \backslash \mathrm{Cl}_{\beta X}(Z) \mid Z\right.$ is a zero-set in $\left.X\right\}$. Again it suffices to show that $\mathscr{U}$ satisfies the hypothesis of Corollary 3 . This proceeds as in Theorem 6 up to the point of showing that if $F \subset G$ are zerosets of $X$ such that $\mathrm{Cl}_{\beta X}(F) \cap(\beta X \backslash X)=\mathrm{Cl}_{\beta X}(G) \cap(\beta X \backslash X)$, then $G \backslash F$ is relatively compact. If not, then there is a $y \in \mathrm{Cl}_{\beta X}(G \backslash F) \cap(\beta X \backslash X)$. Since $X$ is realcompact, every point in $\beta X \backslash X$ is contained in a zeroset disjoint from $X$. Thus there is a $g \in C(\beta X)$ so that $g(y)=0$ and $g(x)>0$ for $x \in X$. If $h(x)=1 / g(x)$, for each $x \in X$, then $h$ is a continuous function on $X$ that is unbounded on $G \backslash F$. Consequently [8,1.20], there is a closed, $C$-embedded copy of $\mathbf{N}$, $D \subset G \backslash F$. Now $\varnothing \neq \mathrm{Cl}_{\beta X}(D) \cap(\beta X \backslash X) \subset \mathrm{Cl}_{\beta X}(G) \cap(\beta X \backslash X)$. Since $D$ is $C$-embedded and disjoint from the zeroset $F$, we have $[8,1.18] \mathrm{Cl}_{\beta X}(D) \cap$ $\mathrm{Cl}_{\beta X}(F)=\varnothing$. This contradiction implies that $G \backslash F$ must be relatively compact. The remainder follows as in Theorem 6.

As the referee points out, a paracompact space is realcompact if no measurable cardinals exist. This is a consequence of Shirota's theorem [8, p. 229]. Thus Theorem 7 is "almost" stronger than Theorem 6.

This of course leaves as an interesting question whether or not the hypothesis that $X$ is paracompact, or realcompact, can be replaced by the hypothesis that $X$ is normal. It certainly seems that this should be the case. More generally, we have the following

Unresolved question. Are all compactifications of a locally compact space ESHcompactifications?

As was indicated in the introduction, the definition of ESH-compactifications generalizes the notion of a singular compactification. In Example 1 we indicated that all singular and weakly singular compactifications are ESH-compactifications. The following theorems explore the converse relationship and characterize singular and weakly singular compactifications among ESH-compactifications.

Theorem 8. Let $\alpha X=X \cup_{\pi} K$ be an ESH-compactification, where $\pi: \mathscr{B} \rightarrow \mathscr{N}$. Suppose $\mathscr{B}$ is closed with respect to finite (unions and) intersections. Then $\alpha X$ is singular if $\pi$ satisfies the following:

(1) $\pi(K)=X$;

(2) $\pi\left(U_{1} \cup U_{2}\right)=\pi\left(U_{1}\right) \cup \pi\left(U_{2}\right)$ for each $U_{1}, U_{2} \in \mathscr{B}$;

(3) $\pi\left(U_{1} \cap U_{2}\right)=\pi\left(U_{1}\right) \cap \pi\left(U_{2}\right)$ for each $U_{1}, U_{2} \in \mathscr{B}$. 
Proof. It will suffice, as indicated in Example 1, to show that there is a retraction $r: \alpha X \rightarrow \alpha X \backslash X=K$. For each $x \in \alpha X$ define

$$
\mathscr{U}_{x}=\{U \in \mathscr{B} \mid x \in U \cup \pi(U)\} \text {. }
$$

It is easy to see that for each $x, \mathscr{U}_{x}$ is a nonempty collection that, by property 3 , is closed under finite intersections. Since $K$ is compact, $\bigcap_{U \in \mathscr{U}_{x}} \mathrm{Cl}_{K}(U) \neq \varnothing$. In fact, $\bigcap_{U \in \mathscr{U}_{x}} \mathrm{Cl}_{K}(U)$ consists of a single point of $K$. To see this suppose that both $s$ and $t$ belong to $\bigcap_{U \in \mathscr{U}_{x}} \mathrm{Cl}_{K}(U)$. Choose $V, W \in \mathscr{B}$ so that $s \in V \backslash \mathrm{Cl}_{K}(W), t \in V \backslash \mathrm{Cl}_{K}(W)$, and $V \cup W=K$. This is possible since $K$ is compact. Now $(V \cup \pi(V)) \cup(W \cup \pi(W))=\alpha X$ so, without loss of generality, we may assume that $x \in V \cup \pi(V)$. However, this implies that $t \in \mathrm{Cl}_{K}(V)$, which is a contradiction. With this in mind we may define $r(x)$ to be the unique element of $\bigcap_{U \in \mathscr{U}_{x}} \mathrm{Cl}_{K}(U)$. If $x \in K$, then it is clear that $r(x)=x$. It remains only to show that $r$ is continuous. Suppose $V$ is open in $K$ and $y \in r^{-1}(V)$. From this we clearly have that $\bigcap_{U \in \mathscr{U}_{y}} \mathrm{Cl}_{K}(U) \subset V$, and so, by compactness, there exist finitely many $U_{1}, U_{2}, \ldots, U_{n}$ such that $\bigcap_{l=1}^{n} \mathrm{Cl}_{K}\left(U_{l}\right) \subset V$. Let $U=U_{1} \cap U_{2} \cap \cdots \cap U_{n}$. Then $U \in \mathscr{B}$ and $\mathrm{Cl}_{K}(U) \subset V$. So for each $z \in U \cup \pi(U)$ we have $\bigcap_{U \in \mathscr{U}_{z}} \mathrm{Cl}_{K}(U) \subset V$. Hence $y \in U \cup \pi(U) \subset r^{-1}(V)$, so that $r$ is continuous.

If $\pi$ satisfies the hypotheses of the above theorem, one has $X \cup_{\pi} K=X \cup_{f} K$, where $f=\left.r\right|_{X}$. Also it can be easily proved that, for every $U \in \mathscr{B}$, one has $f^{-1}(U) \subset \pi(U) \subset f^{-1}\left(\mathrm{Cl}_{K}(U)\right)$. The following example shows that $\pi$ can be different from $f^{-1}$.

Example. Let $X=I \times \mathbf{N}$, with the discrete topology (where $I$ is the real closed interval $[0,1]$ and $\mathbf{N}$ is the set of the natural numbers). Let $K=I$ with the ordinary topology, $f: X \rightarrow K$ the set-theoretic projection. Then $f$ is a singular map. Let $\mathscr{B}$ be the family of the open subsets of $K$ with a finite number of connected components. $\mathscr{B}$ is a basis for the open subsets of $K$ and it is closed with respect to finite unions and intersections. For every $U \in \mathscr{B}$, put

$$
E(U)=\left\{x \in K \mid \exists \delta \in \mathbf{R}^{+}:(x, x+\delta) \subset U \text { or } x=1 \in U\right\} .
$$

One has $U \subset E(U) \subset \bar{U}$. It is easy to see that $E(\varnothing)=\varnothing, E(K)=K$, $E(U \cap V)=E(U) \cap E(V)$, and $E(U) \cup E(V) \subset E(U \cup V)$. Let $x \in E(U \cup V)$. If $x=1 \in U \cup V$, then $x \in E(U) \cup E(V)$. Otherwise, let $(x, x+\delta) \subset U \cup V$, with $\delta \in \mathbf{R}^{+}$. Let $U=\bigcup_{k=1}^{n} I_{k}, V=\bigcup_{h=1}^{m} J_{h}$, where the $I_{k}$ 's, $J_{h}$ 's are open intervals. Then $(x, x+\delta)=\left(\bigcup\left((x, x+\delta) \cap I_{k}\right)\right) \cup\left(\bigcup\left((x, x+\delta) \cap J_{h}\right)\right)$. So we have written $(x, x+\delta)$ as a finite union of intervals that are contained in $U$ or in $V$. One of them must be of the form $(x, x+\varepsilon)$ and this implies $x \in E(U)$ or $x \in E(V)$. This proves the inclusion $E(U \cup V) \subset E(U) \cup E(V)$. If we put $\pi(U)=f^{-1}(E(U))$ for every $U \in \mathscr{B}$, then $\pi$ satisfies the hypotheses of the above theorem. Let $r: X \cup_{\pi} K \rightarrow K$ be the retraction induced by $\pi$ and let $(x, t) \in X$. If $(x, t) \in \pi(U)$ then $f(x, t)=x \in E(U) \subset \mathrm{Cl}_{K}(U)$. Then $f(x, t) \in \bigcap_{(x, t) \in \pi(U)} \mathrm{Cl}_{K}(U)=\{r(x, t)\}$, that is, $\left.r\right|_{X}=f$. But, in general, $\pi(U) \backslash f^{-1}(U)$ is nonempty and not (relatively) compact.

We can now use Theorem 8 to characterize weakly singular compactifications.

Proposition 9. Let $\alpha X=X \cup_{\pi} K$ be an ESH-compactification, where $\pi: \mathscr{B} \rightarrow$ $\mathscr{N}$. Suppose $\mathscr{B}$ is closed with respect to finite (unions and) intersections. Then 
$\alpha X$ is weakly singular if there is a fixed compact set $F \subset X$ so that $\pi$ satisfies the following.
(1) $X \backslash \pi(K) \subset F$;
(2) $\pi\left(U_{1} \cup U_{2}\right) \triangle\left(\pi\left(U_{1}\right) \cup \pi\left(U_{2}\right)\right) \subset F$ for each $U_{1}, U_{2} \in \mathscr{B}$;
(3) $\pi\left(U_{1} \cap U_{2}\right) \triangle\left(\pi\left(U_{1}\right) \cap \pi\left(U_{2}\right)\right) \subset F$ for each $U_{1}, U_{2} \in \mathscr{B}$.

Proof. Let $W$ be a relatively compact open neighbourhood of $F$ and let $T=$ $X \backslash W$. For every $U \in \mathscr{B}$ put $\pi_{1}(U)=\pi(U) \cap T$. Let $\mathscr{N}^{\prime}$ be the family of the subsets $V$ of $T$, open in $T$ and such that $V=\varnothing$ or $\mathrm{Cl}_{T}(V)$ is not compact. One has $\pi_{1}(U) \in \mathscr{N}^{\prime}, \forall U \in \mathscr{B}$. In fact, if $U \neq \varnothing$ then one has $\pi(U) \subset \pi_{1}(U) \cup W$, so $\mathrm{Cl}_{T}\left(\pi_{1}(U)\right)=\mathrm{Cl}_{X}\left(\pi_{1}(U)\right)$ cannot be compact. Then $\pi_{1}: \mathscr{B} \rightarrow \mathscr{N}^{\prime}$ is an ESH and satisfies the hypotheses of the above theorem. Therefore, we can construct the compastification $T \cup_{\pi_{1}} K$ and there exists a retraction $r_{1}$ from $T \cup_{\pi_{1}} K$ to $K$. The equality

$$
U \cup\left(\pi_{1}(U) \backslash G\right)=(U \cup(\pi(U) \backslash G)) \cap(T \cup K),
$$

where $U \in \mathscr{B}$ and $G$ is a compact subset of $T$, shows that the topology of $T \cup K$ as a subspace of $X \cup_{\pi} K$ is finer than the topology of $T \cup_{\pi_{1}} K$. Since both topologies make $T \cup K$ compact, they are equal. But $T \cup K$ is a neighbourhood of $K$ in $X \cup_{\pi} K$, so the conclusion follows.

\section{AN APPLICATION}

In this section we will illustrate the use of these constructions to prove a theorem of Parovičenko [9]. It is this theorem that originally motivated the definition of an ESH. It is a context where singular mappings are of little use. The set of natural numbers will be denoted by $\mathbf{N}$. For every subset $A$ of $\mathbf{N}$, let $A^{*}$ denote the set $\mathrm{Cl}_{\beta \mathbf{N}}(A) \backslash \mathbf{N}$. Sets of this form are clopen in $\mathbf{N}^{*}=\beta \mathbf{N} \backslash \mathbf{N}$ and form a basis for the topology on $\mathbf{N}^{*}$. We denote the collection of these sets by $\mathrm{CO}\left(\mathbf{N}^{*}\right) . \operatorname{CO}\left(\mathbf{N}^{*}\right)$ is a Boolean algebra with the operations $A^{*} \vee B^{*}=A^{*} \cup B^{*}$ and $A^{*} \wedge B^{*}=A^{*} \cap B^{*}$. The properties of these sets are well known and can be found in [11, p. 73]. We will make liberal use of these properties. In particular, $A^{*}=B^{*}$ iff $A \triangle B$ is finite (compact). It is known that if $\mathscr{E}$ is a Boolean algebra of cardinality less than or equal to $\aleph_{1}$, then there exists a Boolean algebra homomorphism $\sigma$ from $\mathscr{E}$ into $\mathrm{CO}\left(\mathbf{N}^{*}\right)$ [11, p. 82]. Let $K$ be a compact Hausdorff space and let $\mathrm{RO}(K)$ denote the collection of regular open subsets of $K$. It is known [10, p. 5] that $\mathrm{RO}(K)$ is a Boolean algebra with the operations given by $U \vee V=(\overline{U \cup V})^{\circ}$ and $U \wedge V=U \cap V$. In the following theorem the Continuum Hypothesis is assumed. We denote by $w(K)$ the weight of $K$.

Theorem 10 (Parovičenko). Let $K$ be a compact Hausdorff. Then $w(K) \leq \aleph_{1}$ iff $K$ is a remainder of $\mathbf{N}$.

Proof. If $K$ is a remainder of $\mathbf{N}$, then $K$ is the continuous image of $\mathbf{N}^{*}$. Since such a mapping must be perfect, $w(K) \leq \aleph_{1}$. Conversely suppose that $w(K) \leq \aleph_{1}$. Let $\mathscr{B}$ be a basis for $K$ with cardinality less than or equal to $\aleph_{1}$. We may suppose without loss of generality that $\mathscr{B}$ is closed under finite unions. For $U \in \mathscr{B}$ let $U^{\prime}=(\bar{U})^{\circ}$ and let $\mathscr{B}^{\prime}=\left\{U^{\prime} \mid U \in \mathscr{B}\right\} \subset \operatorname{RO}(K)$. Let $\mathscr{E}$ be the smallest Boolean subalgebra of $\mathrm{RO}(K)$ containing $\mathscr{B}^{\prime}$. Note that $|\mathscr{E}| \leq \aleph_{1}$. Define a mapping $j: \mathscr{B} \rightarrow \mathscr{E}$ by $j(U)=U^{\prime}$. Now $j(K)=K$ and 
since $\bar{U}=\overline{(\bar{U})^{\circ}}$ for open sets $U$, we have $j\left(U_{1} \cup U_{2}\right)=j\left(U_{1}\right) \vee j\left(U_{2}\right)$. Further, if $U_{1}, U_{2} \in \mathscr{B}$ satisfy $\bar{U}_{1} \cap \bar{U}_{2}=\varnothing$, then $j\left(U_{1}\right) \cap j\left(U_{2}\right)=\left(\bar{U}_{1}\right)^{\circ} \cap\left(\bar{U}_{2}\right)^{\circ}=$ $\left(\bar{U}_{1} \cap \bar{U}_{2}\right)^{\circ}=\varnothing$. For $A^{*} \subset \mathbf{N}^{*}$ let $\left[A^{*}\right]=\left\{B \subset \mathbf{N} \mid A^{*}=\mathrm{Cl}_{\beta \mathbf{N}}(B) \backslash \mathbf{N}\right\}$. Let $\varepsilon$ be a choice function on the collection of equivalence classes [ $\left.A^{*}\right]$ satisfying $\varepsilon([\varnothing])=\varnothing$ and let $i: \operatorname{CO}\left(\mathbf{N}^{*}\right) \rightarrow 2^{\mathbf{N}}$ be given by $i\left(A^{*}\right)=\varepsilon\left(\left[A^{*}\right]\right)$. Now the mapping $\pi=i \circ \sigma \circ j$ is an ESH. Hence $K$ is a remainder of $\mathbf{N}$.

\section{REFERENCES}

1. G. L. Cain, Jr., R. E. Chandler, and G. D. Faulkner, Singular sets and remainders, Trans. Amer. Math. Soc. 268 (1981), 161-171.

2. A. Caterino and M. C. Vipera, Singular compactifications and compactification lattices, Rend. Circ. Mat. Palermo (2) Suppl. 24 (1990), 299-309.

3. Richard E. Chandler, Hausdorff compactifications, Marcel Dekker, New York, 1976.

4. R. E. Chandler and G. D. Faulkner, Singular compactifications: the order structure, Proc. Amer. Math. Soc. 100 (1987), 377-382.

5. R. E. Chandler, G. D. Faulkner, J. P. Guglielmi, and M. Memory, Generalizing the Alexandroff-Urysohn double circumference construction, Proc. Amer. Math. Soc. 83 (1981), 606-608.

6. W. W. Comfort, Retractions and other continuous maps from $\beta X$ onto $\beta X \backslash X$, Trans. Amer. Math. Soc. 114 (1965), 843-847.

7. G. D. Faulkner, Compactifications whose remainders are retracts. Proc. Amer. Math. Soc. 103 (1988), 984-988.

8. L. Gillman and M. Jerison, Rings of continuous functions, New York, 1960.

9. I. I. Parovičenco, A universal bicompact of weight $\aleph$, Dokl. Acad. Nauk SSSR 150 (1963), 36-39; English transl., Soviet Math. Dokl. 4 (1963), 592-595.

10. R. Sikorski, Boolean algebras, Springer-Verlag, New York, 1964.

11. R. C. Walker, The Stone-Čech compactification, Springer-Verlag, New York, 1974.

12. H. Wallman, Lattices and topological spaces, Ann. of Math. (1) 39 (1938), 112-126.

(A. Caterino and M. C. Vipera) Dipartimento di Matematica, Universita, Via Vanvitelli 1-06100 Perugia, Italy

E-mail address: CATVIP AT IPGUNIV, BITNET

(G. D. Faulkner) Department of Mathematics, North Carolina State University, Raleigh, North Carolina 27695-8205

E-mail address: GDF AT GDFSUN.MATH.NCSU.EDU 